\section{New Horizons in Electrochemical Science and Technology \\ National Materials Advisory Board, Commission on Engineering and Technical Systems, National Research Council \\ (National Academy Press, 1986)}

This book reviews the status of current knowledge of electrochemical science and technology and makes recommendations for future $R \& D$ in this multidisciplinary field. The review resulted from a request by the Office of Conservation and Renewable Energy of the Department of Energy that the National Research Council, through the National Materials Advisory Board, assess electrochemical science and technology and recommend opportunities and priorities in $R \& D$ directed toward energy conservation. The committee formed to accomplish this assessment consisted of members of academia and industry, and its task was to assess electrochemical R\&D that could lead to major gains in materials and energy conservation. Its members also directed the efforts of two panels, one to report on corrosion, the other on in situ characterization of electrochemical processes.

The first chapter presents an overview of conclusions and recommendations. Some of these conclusions: (1) major opportunities for new products and processes based on electrochemistry exist outside of conventional electrochemical industries; (2) rapid evolution will soon occur in several critical areas of basic electrochemical science, supporting significant new technological developments; (3) advances in instrumental techniques are making possible major gains in understanding electrochemical systems; (4) a multidisciplinary approach will help solve many problems in electrochemical technologies; (5) the United States needs to improve its ability to transform electrochemical research into products; and (6) the goal of federal support to electrochemical technology should be to foster a broad science and technology base.

Chapter 2 describes many ways in which electrochemical phenomena underpin related technologies, and it argues that the electrochemical field is now in a position to make major advances in science and technology. Chapter 3 considers the socioeconomic contributions of electrochemical technology. A discussion of financial support by the federal government is given in Chapter 4, which states that corrosion and advanced energy conversion devices have been the primary recipients. Opportunities in particular technologies is the subject of Chapter 5, which denotes those industries in which advances in electrochemical devices and processes will have a significant economic impact in less than ten years. The subject of corrosion is covered in this chapter. Chapter 6 outlines opportunities in key technical areas which could lead to new products and processes in the long term of more than ten years, and Chapter 7 addresses the effectiveness of the United States' university community in providing education and training in electrochemical science and engineering.

This book offers the specialist a comprehensive overview of the contributions that electrochemical science and technology are making to modern society. More importantly, it cites areas needing additional development, and it identifies opportunities for exploiting electrochemical processes for extending or developing new technologies.

Reviewer: Ronald Diegle is supervisor of the Corrosion Division, Sandia National Laboratories, Albuquerque, NM.

\section{High Speed Electronics \\ $B$. Kallback and $H$. Beneking \\ (Springer-Verlag, 1987)}

This small volume contains a collection of papers given at the High Speed Electronics Conference (a satellite conference to the International Conference on the Physics of Semiconductors) held at Saltjobaden near Stockholm in August 1986. The conference volume covers basic physical, device-related phenomena and concentrates on III-V compounds. Subjects such as hot electrons, quantum wells, contemporary high speed devices, and some topics in optoelectronics are included.

The newly emerging field of hot electron devices (which usually operate at low temperatures) and ballistic transport is covered extensively by five groups. The THETA device, a promising fast heterojunction device, was exploited as a tool to investigate hot electron transport. This investigation led, for the first time, to a direct observation of ballistic transport in heavily doped GaAs. As a result of the ballistic transport, quantum interference effects were evident. A resonant tunneling emitter was also incorporated in the THETA device to make a multifunction device with a negative differential transconductance. The PDBT, a similar homojunction hot electron device, was also used to study the dynamics of hot electron transport. A newer member of the family, an induced-base device (undoped base and electrons are induced by an ap- plied voltage), was shown to have a current transfer ratio of 0.96 for a $10 \mathrm{~nm}$-thick base, the highest ever reported so far.

Continuing in the vane of perpendicular current transport through layers, two more topics are reported: a novel HBT that incorporates a resonant tunneling structure in the base, and tunneling through uncostumerily thick and low barriers. In particular, the HBT exhibited a room temperature negative differential transfer ratio with possible applications as a multistage device (similar to the modified hot electron device).

Hot electrons which were produced via electron transport parallel to heterojunction interfaces and subsequently real space transferred were exploited in the novel NERFET device. The same effect, useful in the NERFET, was found to be a nuisance (an undesired gate current) in the GaAs-Gate FET device, a device similar to the MODFET but with a $n+\mathrm{GaAs}$ gate (instead of a metal gate).

On the theory side of transport one can find numerical and Monte-Carlo-based calculations of transport in quantum wells and in $n^{+} n^{-} n+$ short channels, short enough to be in the quasi ballistic regime. Implications on velocity overshoot in short channels and on the operation of MODFET type devices are drawn by a few papers.

In the field of optoelectronics two main themes are addressed. The first covers the dynamics of electron motion in quantized structures, such as the evolution of electron mobilities, electron-hole drag effects, and excitonic behavior. The second covers newly emerging techniques to measure device speed by using extremely short optical pulses. The pulses were used to generate very short electrical pulses via the electro-optic effect, photo-emission, and the use of Austin switches. These techniques allow measuring much faster device speed relative to conventional techniques.

MODFETs, HBTs, Schottky detectors, and optical modulators are only briefly discussed.

This relatively small three-day conference succeeded in covering very well most of the main hot topics in devicerelated quantum wells and heterojunctions. The reader might find this volume timely and informative.

Reviewer: $M$. Heiblum, manager of the Microstructure Physics Group, IBM T.J. Watson Research Center, NY, has special interests in physics of hot electrons, quantum effects, and ultrahigh speed devices. 